\title{
I livelli di colesterolo LDL sono associati alla secrezione insulinica
}

\author{
Maria Chiara Zatelli ${ }^{1}$ Irene Gagliardi ${ }^{1} \cdot$ Marta Bondanelli $^{1}$
}

Accettato: 19 maggio 2021 / Pubblicato online: 14 settembre 2021

(c) The Author(s) 2021

\section{Commento a:}

\section{Low-density lipoprotein cholesterol is associated with} insulin secretion.

\section{Dannecker, R. Wagner, A. Peter, J. Hummel,}

A. Vosseler, H.-U. Häring, A. Fritsche, A.L. Birkenfeld, N. Stefan, M. Heni.

\section{J Clin Endocr Metab (2021) 106(6):1576-1584}

La terapia ipolipemizzante con statine rappresenta un pilastro nella prevenzione degli eventi cardiovascolari. Recenti meta-analisi hanno evidenziato che la terapia con statine si associa a un aumento dell'incidenza di nuovi casi di diabete mellito di tipo 2 (DM2) [1]. Non è chiaro, però, se i pazienti valutati siano già predisposti allo sviluppo di DM2 oppure se la terapia con statine causi questo evento. In questo studio, quindi, gli autori valutano l'associazione fra i livelli di LDL colesterolo, i meccanismi patogenetici del DM2 e la secrezione insulinica [2] in una coorte di più di tremila pazienti ad alto rischio di DM2. Lo studio ha evidenziato l'assenza di associazione fra i livelli di LDL colesterolo e i livelli di glucagone a digiuno o dopo OGTT. D'altra parte, è stata evidenziata un' associazione positiva fra i livelli di LDL colesterolo e gli indici di secrezione insulinica basati sul peptide $\mathrm{C}$ e un'associazione negativa fra i livelli di LDL colesterolo e l'indice insulinogenico. Gli autori concludono che, dal momento che il peptide $\mathrm{C}$ riflette la secrezione di insulina indipendentemente dalla clearance epatica, i loro risultati indicano la presenza di una minore secrezione di insulina in caso di livelli minori di LDL colesterolo. Questo riscontro

$\triangle$ M.C. Zatelli

maria.chiara.zatelli@unife.it

1 Sezione di Endocrinologia e Medicina Interna, Dipartimento di Scienze Mediche, Università degli Studi di Ferrara, Ferrara, Italia potrebbe spiegare il peggioramento del controllo glicemico in risposta a farmaci che riducono i livelli di colesterolo.

Questo studio risulta essere significativo perché l'utilizzo delle statine come farmaco ipolipemizzante è estremamente frequente nei pazienti con sindrome metabolica, che sono altrettanto a rischio di sviluppare disturbi dell'omeostasi glucidica.

Il riscontro, forse, più rilevante di questo studio è rappresentato dal fatto che i livelli di LDL colesterolo sono risultati correlati alla secrezione e alla clearance dell'insulina, ponendo le basi per nuovi orizzonti fisiopatologici per le malattie del metabolismo glucidico e lipidico, andando a sottolineare ancora una volta la centralità del fegato come organo omeostatico.

Questi dati suggeriscono, inoltre, prudenza nell'uso delle statine in soggetti con disturbi del metabolismo glucidico, che ne potrebbe essere ulteriormente danneggiato.

Funding Note Open access funding provided by Università degli Studi di Ferrara within the CRUI-CARE Agreement.

Nota della casa editrice Springer Nature rimane neutrale in riguardo alle rivendicazioni giurisdizionali nelle mappe pubblicate e nelle affiliazioni istituzionali.

Open Access This article is licensed under a Creative Commons Attribution 4.0 International License, which permits use, sharing, adaptation, distribution and reproduction in any medium or format, as long as you give appropriate credit to the original author(s) and the source, provide a link to the Creative Commons licence, and indicate if changes were made. The images or other third party material in this article are included in the article's Creative Commons licence, unless indicated otherwise in a credit line to the material. If material is not included in the article's Creative Commons licence and your intended use is not permitted by statutory regulation or exceeds the permitted use, you will need to obtain permission directly from the copyright holder. To view a copy of this licence, visit http://creativecommons.org/licenses/by/4.0/. 


\section{Bibliografia}

1. Swerdlow DI, Preiss D, Kuchenbaecker KB et al (for the DIAGRAM Consortium MAGIC Consortium, MAGIC Consortium, InterAct Consortium) (2015) HMG-coenzyme A reductase inhibition, type 2 diabetes, and bodyweight: evidence from genetic analysis and randomised trials. Lancet 385(9965):351-361

2. Matsuda M, DeFronzo RA (1999) Insulin sensitivity indices obtained from oral glucose tolerance testing: comparison with the euglycemic insulin clamp. Diabetes Care 22(9):1462-1470 